\title{
Nondimensional analysis of particle behavior during cross-type optical particle separation
}

\author{
Sang Bok Kim, ${ }^{1}$ Hyung Jin Sung, ${ }^{1, *}$ and Sang Soo Kim ${ }^{1,2}$ \\ 'Department of Mechanical Engineering, Korea Advanced Institute of Science and Technology, \\ Guseong-dong, Yuseong-gu, Daejeon 305-701, South Korea \\ ${ }^{2}$ E-mail: sskim@ @aist.ac.kr \\ *Corresponding author: hjsung@kaist.ac.kr
}

Received 14 May 2009; revised 3 July 2009; accepted 10 July 2009; posted 10 July 2009 (Doc. ID 111393); published 21 July 2009

\begin{abstract}
A nondimensional analysis of particle behavior during cross-type optical particle separation was performed. A new dimensionless number, $S$, was defined as the ratio of the optical force to the viscous drag force, and the effects of varying $S$ on particle motion were examined. For large $S$, the particles undergo acceleration, deceleration, and release as they pass through the laser beam. The retention distance is much longer for large $S$ than for small $S$. In addition, the effects on particle behavior of varying the wavelength of the laser beam, the particle size, and the index of refraction of the particles were investigated. Furthermore, an analytical expression of the retention distance for large $S$ was validated. (C) 2009 Optical Society of America

OCIS codes: $\quad 260.0260,080.0080$.
\end{abstract}

\section{Introduction}

The separation of particles in a microchannel is considered an important technique in various areas, including analytical chemistry, biological cell research, and lab-on-a-chip applications [1-3]. With the development of microfabrication techniques, various methods of particle separation have been developed, such as dielectrophoretic [4,5], magnetophoretic [6,7], hydrophoretic [8,9], and optical separation methods [10-18]. Optical particle separation has some advantages over the other methods: relatively simple structures can be used, resulting in easy to control separation characteristics, and pre- and posttreatments of the medium or separated particles are not required.

The origins of the optical separation method lie in Ashkin's first demonstration of the optical acceleration with a laser beam of glass microspheres in water [19]. Optical forces have subsequently been successfully applied to the trapping and separation of particles. With the advent of fiber optic [16-18] and

0003-6935/09/224291-06\$15.00/0

(C) 2009 Optical Society of America waveguide techniques [20], continuous separation of particles has been achieved. In particular, crosstype optical particle separation, as illustrated in Fig. 1, is well established theoretically and experimentally [15-18]. In cross-type optical particle separation, a laser beam propagates in a direction perpendicular to that of the stream of particles, and the resulting scattering force, which acts on the particles in the direction of laser beam propagation, diverts the particles. Due to the dependence of the scattering force on the properties of the particles, the particles are separated by their passage through the laser beam. Kim et al. [15] first proposed the cross-type optical particle separation method and analyzed the behavior of particles during the separation process. They also derived an analytical expression for the retention distance that can be used to estimate the maximum displacement of particles after their passage through the laser beam. However, their study considered only variations in the optical properties and sizes of the particles and, by making the small particle assumption, ignored the gradient force that acts on the particles in the direction of the intensity gradient of the laser beam. 
In the present study we attempted to generalize this previous theoretical study [15] by ensuring that the governing equations for particle motion during the cross-type optical particle separation are nondimensional. This nondimensional analysis accounts for the gradient force, which has been ignored by previous studies of cross-type optical particle separation [15]. Since the most important external forces exerted on particles during optical particle separation are the viscous drag force and the optical force, the ratio of these two forces can provide insight into particle behavior. In the present study, the effects of varying the ratio of the optical force to the viscous drag force on particle behavior were determined by using this nondimensional form of the particle dynamics equations. In addition, the effects on particle behavior of varying the particle size, the wavelength of the laser beam, the index of refraction of the particles, and the retention distance were also analyzed. Furthermore, the criteria for validity of the simple analytical form for the retention distance derived in the previous study [15] were determined.

\section{Theory}

In the cross-type optical particle separation depicted in Fig. 1, the motions of the particles can be described with the following equations [15]:

$$
\begin{gathered}
m_{p} \frac{\mathrm{d} u_{p}}{\mathrm{~d} t}+6 \pi \mu r_{p}\left(U-u_{p}\right)=F_{g} \\
m_{p} \frac{\mathrm{d} v_{p}}{\mathrm{~d} t}+6 \pi \mu r_{p} v_{p}=F_{\mathrm{s}}
\end{gathered}
$$

where $m_{p}$ is the particle mass, $\mu$ is the dynamic viscosity of the medium, $U$ is the $x$-directional velocity of the fluid flow, $u_{p}$ is the $x$-directional particle velocity, $r_{p}$ is the radius of the particle, and $v_{p}$ is the $y$ directional particle velocity. $F_{g}$ and $F_{s}$ are the gradient and scattering forces, respectively.

The following nondimensional variables were adopted in the present study:

$$
\begin{array}{llll}
u_{p}^{*}=\frac{u_{p}}{U}, & v_{p}^{*}=\frac{v_{p}}{U}, & x^{*}=\frac{x}{\omega_{0}}, & y^{*}=\frac{y}{\omega_{0}} \\
r_{p}^{*}=\frac{r_{p}}{\omega_{0}}, & t^{*}=\frac{U t}{\omega_{0}}, & \omega^{*}=\frac{\omega}{\omega_{0}}, & \lambda^{*}=\frac{\lambda}{\omega_{0}} .
\end{array}
$$

In this definition of the nondimensional variables, $x$ and $y$ are the $x$ - and $y$-directional positions of the particle, respectively; $\omega_{0}$ is the laser beam waist; $t$ is time, $\lambda$ is the wavelength of the laser beam; and $\omega$ is the radius of the laser beam width at an arbitrary $y$ position, which can be expressed as

$$
\omega=\omega_{0} \sqrt{1+\left(\frac{\lambda y}{\pi \omega_{0}^{2}}\right)^{2}}, \quad \omega^{*}=\sqrt{1+\left(\frac{\lambda^{*} y^{*}}{\pi}\right)^{2}} .
$$

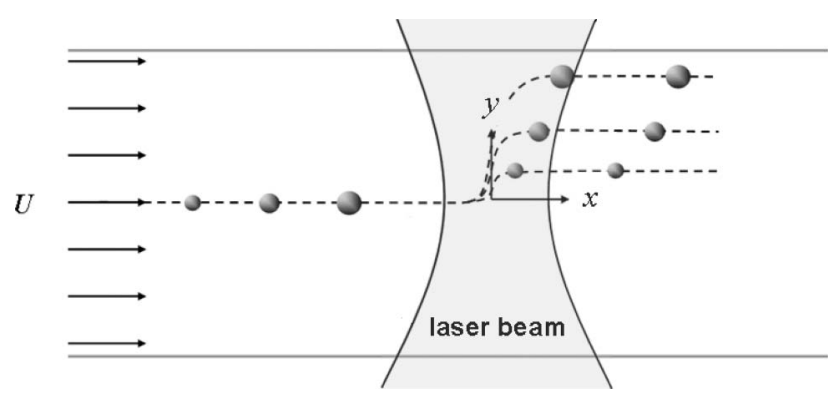

Fig. 1. Schematic diagram of cross-type optical particle separation.

With these nondimensional variables, the equations of motion of a particle during cross-type optical particle separation can be rewritten as

$$
\begin{aligned}
\frac{\mathrm{d} u_{p}^{*}}{\mathrm{~d} t^{*}}= & R\left[\left(1-u_{p}^{*}\right)+S \int_{0}^{2 \pi} \int_{0}^{\pi / 2} \frac{Q_{\mathrm{g}}}{\omega^{* 2}} \exp \left(-\frac{2 \rho^{*}}{\omega^{* 2}}\right) \sin 2 \theta\right. \\
& \times \cos \varphi \mathrm{d} \theta \mathrm{d} \varphi]
\end{aligned}
$$

$\frac{\mathrm{d} v_{p}^{*}}{\mathrm{~d} t^{*}}=R\left[-v_{p}^{*}+S \int_{0}^{2 \pi} \int_{0}^{\pi / 2} \frac{Q_{\mathrm{s}}}{\omega^{* 2}} \exp \left(-\frac{2 \rho^{*}}{\omega^{* 2}}\right) \sin 2 \theta \mathrm{d} \theta \mathrm{d} \varphi\right]$.

In the derivation of the above equations, the intensity profile of the laser beam was assumed to be Gaussian [21,22]. In the integrand, $\rho^{*}$ is the nondimensional distance between the center axis of the laser beam and the center of the particle [21,22], and $Q_{g}$ and $Q_{s}$ are the nondimensional coefficients of the gradient and scattering forces, respectively; $Q_{g}$ and $Q_{s}$ depend only on the relative refractive indices of the particles and the medium [21].

The two nondimensional numbers $R$ and $S$ in Eqs. (ㅁ) and (ㅁ) are obtained from the following equations:

$$
\begin{gathered}
R=\frac{6 \pi \mu r_{p} U}{m_{p} U^{2} / \omega_{0}}=\frac{\text { viscous drag }}{\text { particle inertia }}, \\
S=\frac{\left(n_{0} P / \pi c\right)\left(r_{p}^{2} / \omega_{0}^{2}\right)}{6 \pi \mu r_{p} U}=\frac{\text { optical force }}{\text { viscous drag }} .
\end{gathered}
$$

$R$ is commonly used in particle dynamics equations and is the ratio of the viscous drag force to the inertia of the particle. The dimensionless number $S$ in Eq. (8) is the ratio of the optical force to the viscous drag force. In Eq. (8), $n_{0} P / \pi c$, where $n_{0}$ is the refractive index of the medium, $P$ is the power of the laser beam, and $c$ is the speed of light in free space, represents incident momentum per second from the laser beam [23]. Therefore, the numerator in Eq. (8) is the incident momentum per second on the particle, 
which is equivalent to the optical force on particle by a laser beam having power $P$ and waist $\omega_{0}$. The $\pi$ in the numerator in Eq. (8) is due to the Gaussian intensity distribution in Eqs. (5) and (6). Since $S$ accounts for the effects of the optical force, it governs the trajectories of the particles. In previous studies of cross-type optical particle separation, [15-18] the gradient force was neglected, and a simple relation between the retention distance and the system parameters was derived under the assumption that the particle radius is sufficiently less than the laser beam waist. However, as $S$ increases, the effects of the gradient force can no longer be ignored, and in particular affects the retention distance even when the particle radius is much smaller than the laser beam waist.

\section{Numerical Calculations}

The motions of particles during cross-type optical particle separation can be determined from the governing equations derived in the previous section. In the following numerical calculations, the nondimensional variable $R$, the nondimensional particle radius $r_{p}^{*}$, and the relative index of refraction of the particles $m$ are assumed to be constant:

$$
R=10^{6}, \quad r_{p}^{*}=0.1, \quad m=1.2, \quad \lambda^{*}=0.01 .
$$

The value of $R$ is based on commonly used values for cross-type optical particle separation, i.e., micrometer-sized particles in water with a velocity of the order of $100 \mu \mathrm{m} / \mathrm{s}$. Since the radius of the particles is smaller than the laser beam waist, the nondimensional particle radius was assumed to be 0.1 .

\section{A. Effects of Varying $S$ on the Particle Trajectories}

The trajectories of particles during cross-type optical particle separation were calculated for various values of $S$, as shown in Fig. 2. To analyze the characteristic motions of the particles, the nondimensional $y$ position was normalized by the maximum $y$ displacement $y_{\max }^{*}$. As shown in Fig. $\underline{2}$, the trajectories for $S$ $=0.1$ and 1 have similar behavior. However, for $S=$ 10 , the trajectory deviates significantly from those of the other two cases. According to the definition of $S$, an increase in $S$ corresponds to an increase in the optical force relative to the $x$-directional viscous drag force. Since the gradient force acts along the $x$ direction, the effects of the gradient force become more significant as $S$ increases.

In a more detailed analysis, the $x$-directional particle velocity was calculated as shown in Fig. 3. For small $S$, i.e., $S=0.1$, the $x$-directional particle velocity is constant. However, as $S$ increases, the $x$ directional particle velocity varies. Since the gradient force pulls particles towards the center axis of the laser beam, the particles accelerate when they enter the laser beam and then decelerate after passing through the center axis of the laser beam. This explanation is supported by plotting the $x$ position as a function of time. As shown in Fig. 4 , for $S=0.1$ and

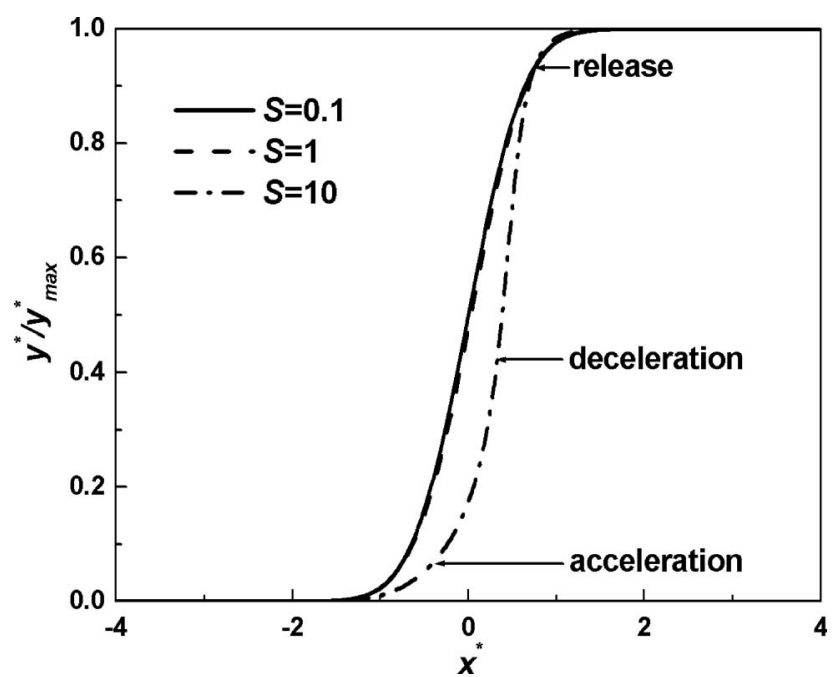

Fig. 2. Normalized trajectories of particles during cross-type optical particle separation for various values of $S$.

1 particles move along the $x$ direction with an almost constant velocity. However, acceleration and deceleration of the particles are clearly detected for $S=10$. When particles are much smaller than the laser beam waist, however, the gradient force should be considered for large $S$.

During acceleration and deceleration, the particles continuously experience the scattering force, which acts in the $y$-direction. Therefore, the particles are continuously moving in the $y$-direction. As the particles move in the $y$-direction, the laser beam width increases according to Eq. (4). Since an increase in the laser beam width reduces the intensity gradient, the gradient force is reduced. When the viscous drag force is larger than the gradient force, the particles are released. The mechanisms of acceleration, deceleration, and release are depicted in Fig. $\underline{5}$.

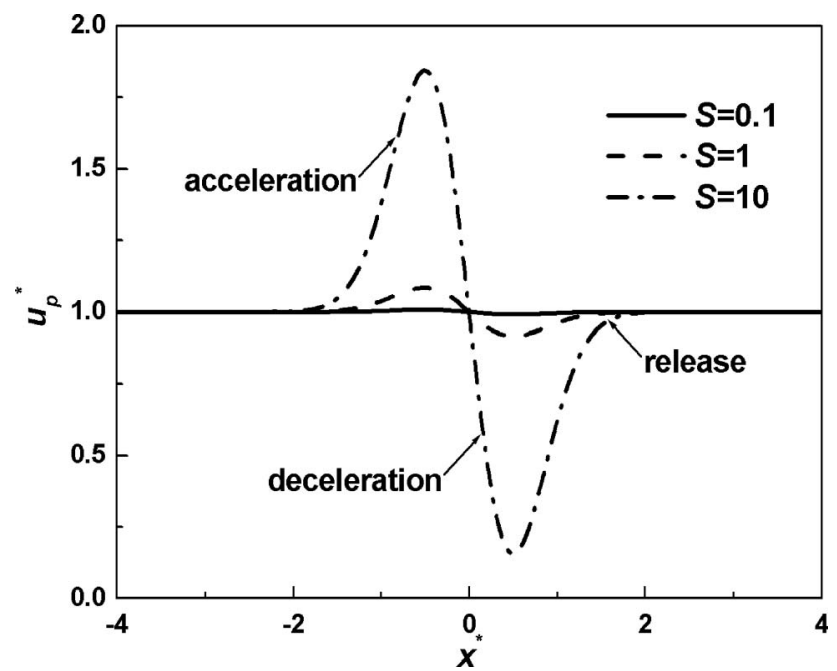

Fig. 3. $x$-directional particle velocities for various values of $S$. As $S$ increases, the particles undergo acceleration, deceleration, and release. 


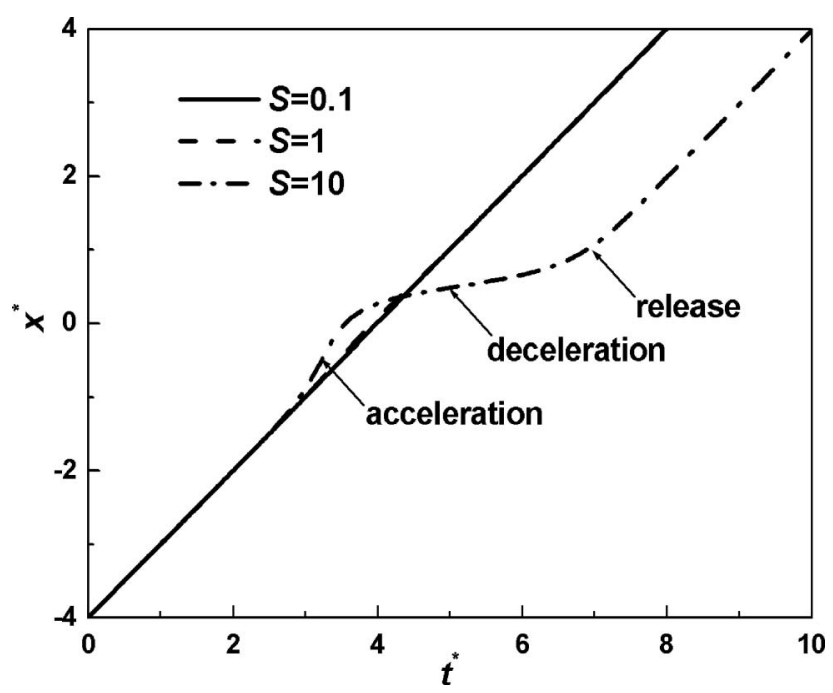

Fig. 4. $x$ positions of particles as a function of time. Due to the deceleration of the particles, the separation time increases for large values of $S$.

\section{B. Effects of Varying $S$ on the Retention Distance}

The retention distance is defined as the maximum displacement of a particle in the direction of laser beam propagation. The retention distance depends on system parameters and the properties of the particles, in particular the particle size and the index of refraction [15]. In our previous study of cross-type optical particle separation, an analytical expression for the retention distance was derived by neglecting the gradient force. However, when $S$ is very large, the effects on particle behavior of the gradient force are significant even when particles are smaller than the laser beam waist.

The retention distance can be expressed in a nondimensional form [15]:

$$
y_{\max }^{*}=S f(m),
$$

where $f(m)$ is a function of the relative index of refraction, i.e., the ratio of the index of refraction of the particles to that of the medium,

$$
f(m)=\sqrt{2 \pi^{3 / 2}} Q^{*}(m) \operatorname{erf}(\sqrt{2}) .
$$

Here, erf is an error function and $Q^{*}$ is the nondimensional coefficient of the scattering force for small particles that depends only on the relative index of refraction $[10,15]$. As shown in Eq. (9), the retention distance has a linear dependence on $\bar{S}$, i.e., the retention distance for $S=10$ is 100 times longer than that for $S=0.1$. However, the nondimensional retention distances calculated from the particle trajectories for $S=10$ and 0.1 are 19.8 and 0.1 , respectively. The retention distance for $S=10$ is then almost 200 times longer than that for $S=0.1$.

In the derivation of Eq. (9), the gradient force was not included. However, when $S$ is large, the effects of the gradient force should be included. Due to the ef-

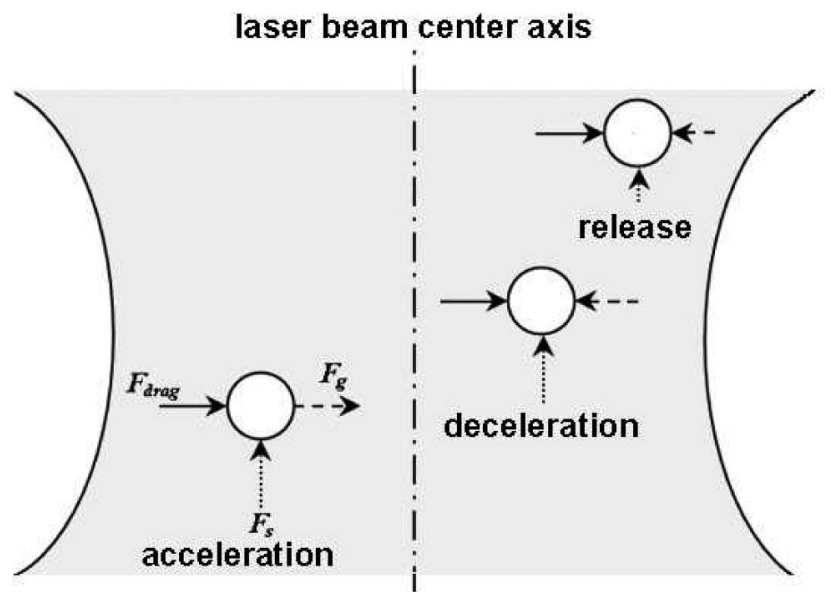

Fig. 5. Mechanisms of acceleration, deceleration, and release of particles.

fects of the gradient force, the linear dependence of the retention distance on $S$ is broken. As shown in Fig. 4 , for large $S$, it takes longer for particles to escape from the laser beam region than $2 \omega_{0} / U$, which is in contrast to the assumption made in our previous study of a constant $x$-directional velocity. Therefore, the particle experiences the scattering force for a longer time and the retention distance increases. Figure $\underline{6}$ shows the variation of the retention distance as a function of $S$. As can clearly be seen in Fig. 6 , for small $S$ the retention distance depends linearly on $S$. However, as $S$ increases, the retention distance deviates from this linear relation.

C. Criteria for a Linear Relation between $S$ and the Retention Distance

In the previous section only the variation of $S$ was considered. However, the optical force also depends on the relative index of refraction, the wavelength of the laser beam, and the particle size. $S$ does not account for the relative index of refraction or the

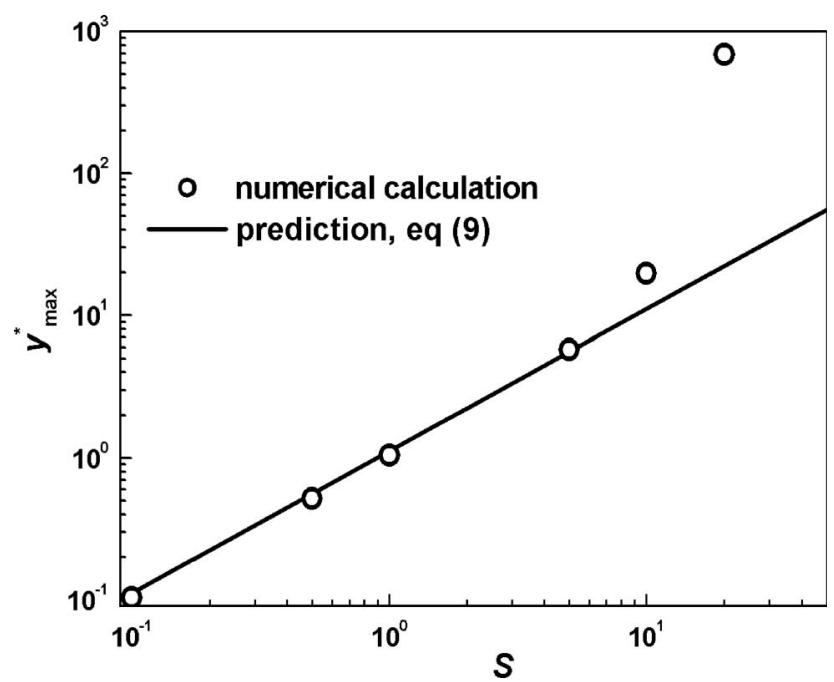

Fig. 6. Retention distance as a function of $S$. For small values of $S$, the retention distance has a linear relation with $S$ but the dependence becomes increasingly nonlinear as $S$ increases. 


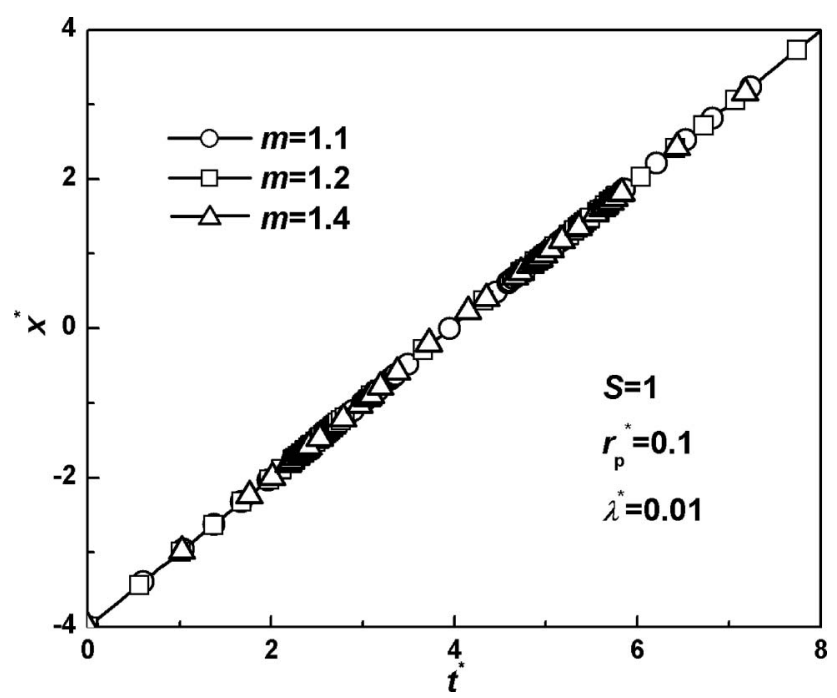

Fig. 7. Effects on the $x$-directional particle velocity of varying the index of refraction of the particles. The variation of the index of refraction of the particles does not affect particle behavior.

wavelength of the laser beam. To account for these effects, the $x$-directional particle velocity was calculated as a function of time for various values of the relative index of refraction and the wavelength of the laser beam. Figures 7 and 8 show the effects on the $x$-directional particle velocity of varying the relative index of refraction and the wavelength of the laser beam, respectively. The range of the relative index of refraction adopted in this calculation covers the cases of biological cells and of standard particles for separation such as polystyrene latex particles suspended in water. Since the laser beam waist is of the order of $100 \mu \mathrm{m}$, the ranges of wavelengths used in these calculations are those of ultraviolet to infrared light. As shown in Figs. 7 and 8 , the acceleration, deceleration, and release of particles do not arise as a result of the variations of the relative index

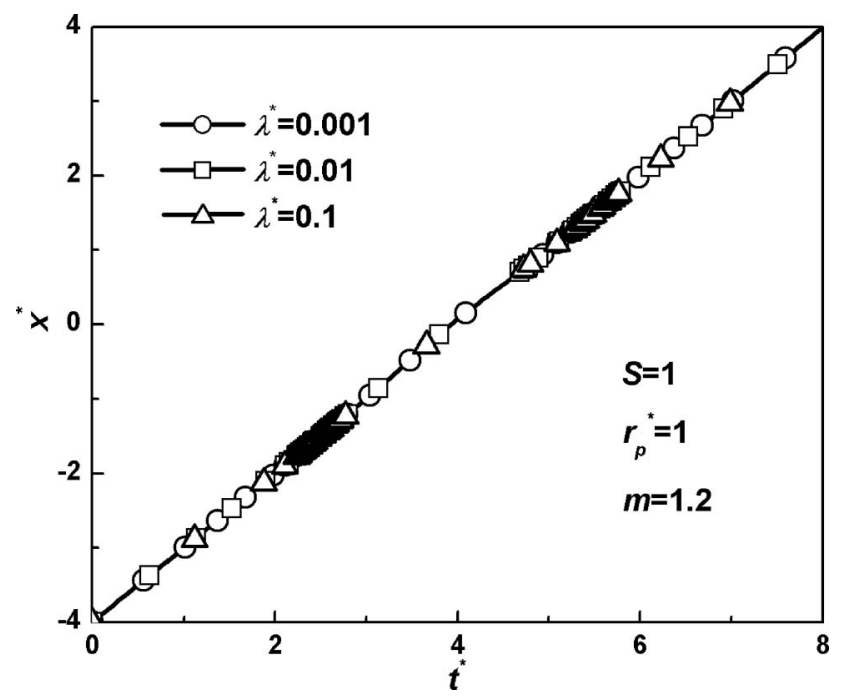

Fig. 8. Effects on the $x$-directional particle velocity of varying the wavelength of the laser beam. The variation of the wavelength of the laser beam does not affect particle behavior.

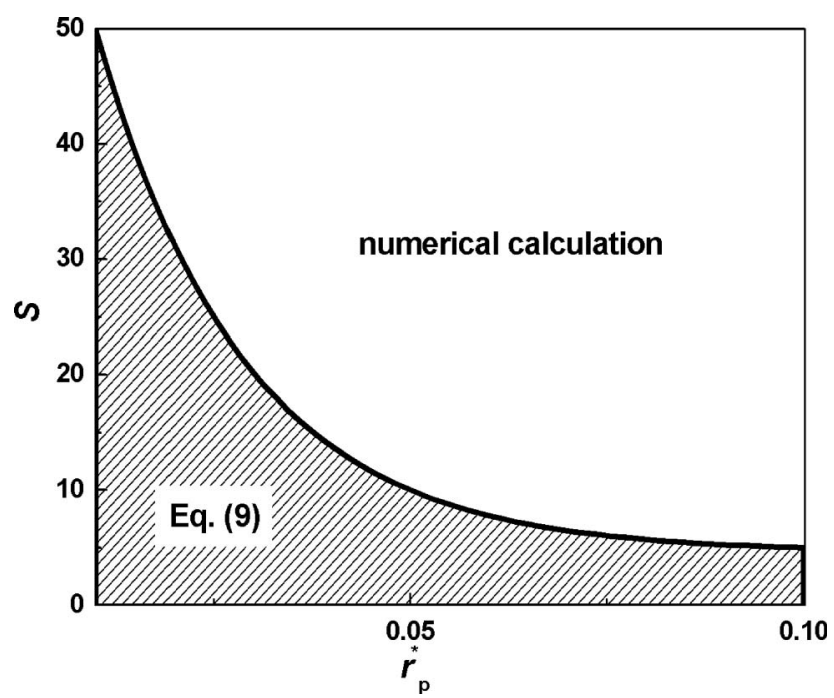

Fig. 9. The shaded region is the region of validity of Eq. (9) $\left(r_{p}^{*} \leq 0.1\right)$. Outside the shaded region, the relation distance is calculated numerically with Eqs. (5) and (6).

of refraction and the wavelength of the laser beam. Further, the linear relation between the retention distance and $S$ is not affected by the relative index of refraction or the wavelength of the laser beam. Therefore, in the common application range, the linear relation holds between the retention distance and $S$.

Finally, the effects on particle behavior of particle size were considered. Although particle size is included in the definition of $S$, particle size can also affect particle behavior because of the functional dependence of $\rho^{*}$ on particle size, as shown in Eqs. (5) and (6). Since Eq. (9) is valid only when the particles are smaller than the laser beam waist, particle sizes $r_{p}^{*} \leq 0.1$ were considered. As shown previously [24], when the particle size $r_{p}^{*} \geq 0.1$, an additional size correction factor is needed, and then Eq. (9) is not valid. To characterize the region of validity of Eq. (9), the upper values of $S$ were determined for various particle sizes. The shaded region in Fig. 9 is the region of validity of Eq. (9). As shown in Fig. $\overline{9}$, as the particle size decreases, the upper limit of $S$ increases. As particle size decreases, the intensity gradient decreases within the dimension of particle diameter. Therefore, the effects of the gradient force are not significant unless $S$ is large. Outside the shaded region in Fig. 9 , the retention distance should be calculated numerically with Eqs. (5) and (6), since the effects of the gradient force cannot be ignored. As long as the experimental conditions lie within the shaded region in Fig. 9, the retention distance can be estimated with Eq. $(\overline{9})$ within $10 \%$ accuracy.

\section{Conclusion}

The effects on particle behavior during cross-type optical particle separation of varying various parameters, namely the wavelength of the laser beam, the particle size, the index of refraction of the particles, and the ratio of the optical force to the viscous drag 
force, have been examined with a nondimensional analysis. Under common application conditions, the ratio of the optical force to the viscous drag force, denoted $S$, is the dominant parameter that governs the behavior of particles. For large $S$, the linear relation between $S$ and the retention distance is no longer valid due to the effects of the gradient force, which was neglected in the previous study. Particles undergo acceleration, deceleration, and release during separation for large $S$. The retention distance can be dramatically increased by increasing $S$, and thus the resolution of the cross-type optical particle separation can be improved. However, increasing the retention distance by increasing $S$ results in a longer separation time. There is thus a trade-off between the resolution of the cross-type optical particle separation and the separation time. The results of the present study can provide guidelines for the design of optimal optical particle separation.

The authors express their gratitude for support through a grant from the Creative Research Initiatives (Center for Opto-Fluid-Flexible Body Interaction) of the Ministry of Education, Science \& Technology/National Research Foundation of Korea (MEST/KOSEF).

\section{References}

1. M. Toner and D. Irimia, "Blood-on-a-chip," Annu. Rev. Biomed. Eng. 7, 77-103 (2005).

2. H. Andersoon and A. van den Berg, "Microfluidic devices for cellomics: a review," Sens. Actuators B 92, 315-325 (2003).

3. J. El-Ali, P. K. Sorger, and K. F. Jensen, "Cells on chips," Nature 442, 403-411 (2006).

4. U. Kim, C.-W. Shu, K. Y. Dane, P. S. Daugherty, J. Y. J. Wang, and H. T. Soh, "Selection of mammalian cells based on their cell-cycle phase using dielectrophoresis," Proc. Natl. Acad. Sci. U.S.A. 104, 20708-20712 (2007).

5. C. M. Das, F. Becker, S. Vermon, J. Noshari, C. Joyce, and P. R. C. Gascoyne, "Dielectrophoretic segregation of different human cell types on microscope slides," Anal. Chem. 77, 27082719 (2005)

6. J. Jung and K.-H. Han, "Lateral-driven continuous magnetophoretic separation of blood cells," Appl. Phys. Lett. 93, 223902 (2008).

7. K. E. McCloskey, J. J. Chalmers, and M. Zborowski, "Magnetic cell separation: characterization of magnetophoretic mobility," Anal. Chem. 75, 6868-6874 (2003).
8. S. Choi, S. Song, C. Choi, and J.-K. Park, "Continuous blood cell separation by hydrophoretic filtration," Lab Chip 7, 1532-1538 (2007).

9. S. Choi and J.-K. Park, "Continuous hydrophoretic separation and sizing of microparticles using slanted obstacles in a microchannel," Lab Chip 7, 890-897 (2007).

10. T. Kaneta, Y. Ishidzu, N. Mishima, and T. Imasaka, "Theory of optical chromatography," Anal. Chem. 69, 2701-2710 (1997).

11. S. J. Hart and A. V. Terray, "Refractive-index-driven separation of colloidal polymer particles using optical chromatography," Appl. Phys. Lett. 83, 5316-5318 (2003).

12. S. J. Hart, A. V. Terray, and J. Arnold, "Particle separation and collection using an optical chromatographic filter," Appl. Phys. Lett. 91, 171121 (2007).

13. R. F. Marchington, M. Mazilu, S. Kuriakose, V. GarcèsChávez, P. J. Reece, T. F. Krauss, M. Gu, and K. Dholakia, "Optical deflection and sorting of microparticles in a near-field optical geometry," Opt. Express 16, 3712-3726 (2008).

14. P. Jákl, T. Čižmár, M. Šerý, and P. Zemánek, "Static optical sorting in a laser interference field," Appl. Phys. Lett. 92, 161110 (2008).

15. S. B. Kim, J. H. Kim, and S. S. Kim, "Theoretical development of in situ optical particle separator: cross-type optical chromatography," Appl. Opt. 45, 6919-6924 (2006).

16. S. B. Kim, S. Y. Yoon, H. J. Sung, and S. S. Kim, "Cross-type optical particle separation in a microchannel," Anal. Chem. 80, 2628-2630 (2008).

17. S. B. Kim, S. Y. Yoon, H. J. Sung, and S. S. Kim, "Resolution of cross-type optical particle separation," Anal. Chem. 80, 60236028 (2008).

18. S. B. Kim, E. Jung, H. J. Sung, and S. S. Kim, "Optical mobility in cross-type optical particle separation,” Appl. Phys. Lett. 93, 044103 (2008).

19. A. Ashkin, "Acceleration and trapping of particles by radiation pressure," Phys. Rev. Lett. 24, 156-159 (1970).

20. A. H. J. Yang, S. D. Moore, B. S. Schmidt, M. Klug, M. Lipson, and D. Erickson, "Optical manipulation of nanoparticles and biomolecules in sub-wavelength slot waveguides," Nature 457, 71-75 (2009).

21. S. B. Kim and S. S. Kim, "Radiation forces on spheres in loosely focused Gaussian beam: ray-optics regime," J. Opt. Soc. Am B 23, 897-903 (2006).

22. R. C. Gauthier and S. Wallace, "Optical levitation of spheres: analytical development and numerical computations of the force equations," J. Opt. Soc. Am B 12, 1680-1686 (1995).

23. A. Ashkin, "Forces of a single-beam gradient laser trap on a dielectric sphere in the ray optics regime," Biophys. J. 61, 569-582 (1992)

24. S. B. Kim, D. K. Song, and S. S. Kim, "Optical differential mobility analyzer for micrometer size colloidal particles: theoretical approach," J. Colloid Interface Sci. 311, 102109 (2007). 